\title{
TENDENCIAS EN MERCADEO SOCIAL (MS)
}

\author{
Aida Ximena León Guatame \\ Institución Universitaria Politécnico Grancolombiano
}

Julio de 2020

\section{RESUMEN}

El Mercadeo Social (MS) son las acciones estratégicas dirigidas a diferentes actores que están directamente relacionados con una situación objeto de cambio, con el fin de lograr cambios de comportamiento o conducta que mejoren la calidad de vida de individuos y comunidades. En su evolución ha sido objeto de investigación de Escuelas como la de Macromarketing y la de pensamiento de investigación transformadora del consumidor (TCR); ha recibido aportes de la teoría de movimiento social, la teoría de red de actores y la teoría de la práctica social y se proyecta una tendencia en la implementación del Marketing Macro-Social para cambiar comportamientos en la sociedad y el Modelo de Mercadeo Social con enfoque Sostenible (MSsS) para planear cambios de comportamiento en la era de los Objetivos de Desarrollo Sostenible (ODS) .

\begin{abstract}
Social Marketing (MS) are strategic actions aimed at different actors directly relationed to a situation that is object change, in order to achieve behavioral changes that improve the quality of life of individuals and communities. The MS it has been the object of research by Schools such as Macromarketing and The transformative consumer research (TCR); He has received contributions from the theory of social movement, the theory of the network of actors and the theory of social practice and a trend is projected in the implementation of Macro-Social Marketing to change behaviors in society and the Social Marketing Model with a focus Sustainable (MSsS) to plan behavior changes in the era of the Sustainable Development Goals (SDG).
\end{abstract}

\section{PALABRAS CLAVES}

Mercadeo Social, Cambio de comportamiento, Sostenibilidad

\section{KEY WORDS}

Social Marketing, Behavior change, Sustainability 


\section{ANTECENDENTES}

Este manuscrito pretende entregar los resultados a la pregunta de investigación ¿Cuáles son los avances de la literatura sobre Mercadeo Social y sus tendencias? En este sentido entiende el Mercadeo Social (en adelante MS) como las acciones estratégicas para propiciar cambios de comportamiento o conducta en las personas para mejorar su calidad de vida, a partir de tomar decisiones informadas que permitan impactar a diferentes actores que están directamente relacionados con la situación objeto de cambio.

El MS ha sido objeto de análisis en diversos sectores, dado su alto potencial para apoyar diversas problemáticas, es así como apoyan la disminución de obesidad o de consumo de alcohol (Fabbrizzi et al., 2017), o de enfermedades de trasmisión sexual, o la inseguridad o los estigmas y promueven la donación de órganos, o la lactancia materna, o la autoestima (Gurrieri et al. 2013).

Su origen fue una consecuencia de la importancia mercantil que se le atribuyó al Marketing por la forma en que estaba cambiando el comportamiento de los consumidores, al incrementar la búsqueda de productos y servicios para satisfacer sus necesidades y las de la sociedad (Lazer, 1969).

Sus precursores fueron Kotler y Zaltman (1971) quienes aplicaron el concepto a la forma de hacer MS en las Organizaciones sin ánimo de lucro. Bajo la aplicación de Marketing Andreasen (1997) se interesó por promover en los nuevos profesionales de ese momento que emplearan la investigación sobre las causas de los comportamientos para hacer uso del MS y atacar la problemática para obtener los resultados esperados.

Esto en razón a que la publicidad social se hacía menos efectiva que las intervenciones de MS porque utilizaban menos criterios de referencia social (Kubacki et al., 2015). Es así como la Publicidad Social actuaba bajo esquemas preconcebidos de forma generalizada sin comprender el contexto que enmarca las conductas y lanzaba campañas de sensibilización con bajo soporte de investigación sobre valores, actitudes, creencias e influenciadores en contextos particulares. Confundiendo así el MS con la forma de comunicar (Andreasen, 1994).

A pesar que el propósito de Andreasen evolucionó en Europa y Estados Unidos, los cambios de comportamiento seguían siendo dirigidos hacia el individuo, desconociendo que los comportamientos 
son el resultado de interacciones con otras personas que cumplen otros roles o sencillamente facilitan la ocasión no deseada (Brennan et al., 2015) y de esta manera se abrió el prospecto de impacto de MS hacia otros niveles.

En cuanto a la clasificación de MS, Kotler y Zaltman (1971) catalogaron el MS en función de su resultado social como Altruista cuando se hacía voluntariado para organizaciones benéficas, o de Salud Personal si promovía actividades para mejorar la salud física o comportamientos de mejora social como el apoyo al cambio climático; posteriormente Kotler y Roberto (1989) propusieron un esquema basado en la tangibilidad del producto de MS, porque algunas causas son menos tangibles que otras por requerir el uso de elementos para lograr resultados y requieren un enfoque más multifacético. La clasificación empleada recientemente es el más difundido como es el MS de buenas causas, el MS Corporativo para promover acciones de cambio de comportamiento en busca de mejorar la calidad de vida de las comunidades y el MS de Causa que promueve esfuerzos de empresas en conjunto con sus clientes para apoyar causas sociales (Kotler \& Lee, 2004).

\section{FUNDAMENTO TEÓRICO}

El MS ha sido analizado por la Escuela del Macromarketing, la cual entiende que el Marketing está inmerso en un sistema de mercado en el cual su interés es la productividad económica y la igualdad social dentro de este sistema, para ello evalúa los efectos del MS en el cambio de comportamiento (eficiencia y eficacia) y su proceso (equidad y sostenibilidad), pero sus consecuencias pueden ser inequitativas e injustas y puede empoderar al consumidor sin lograr una transformación social justa y equitativa (Daellenbach \& Parkinson, 2017).

De otra parte, la Escuela de pensamiento de investigación transformadora del consumidor (TCR) considera a la audiencia objetivo como los transformadores que dan vida a su propia vida, por lo que el cambio social se produce a través de acciones participativas y aprendizajes comunitarios significativos y de valor como consecuencia de la confianza, el intercambio y la colaboración entre todas las partes interesadas. Busca ideas que puedan ser de beneficio directo, para ello examina problemas y oportunidades significativas para los consumidores, analiza las soluciones de evolución automática que experimentan y por las cuales son capaces de generar y empodera a las personas y las comunidades para encontrar soluciones sostenibles que puedan ser la base duradera para una 
sociedad social efectiva y eficiente con programas de marketing, políticas e intervenciones, así genera conocimiento de las experiencias, aspiraciones y capacidades de resolución de problemas de las personas desde el respeto, la defensa y la mejora de la condición humana (Saunders et al., 2015).

En este mismo sentido el Enfoque de la Capacidad reconoce lo que puede hacer y ser cada persona a partir de las oportunidades reales disponibles y es muy importante la libertad de elección y otorga una alta responsabilidad al MS de apoyar a la heterogeneidad y la diversidad de los comportamientos "socialmente buenos". Examinan las habilidades para promover un conjunto de oportunidades, en las que los individuos pueden o no actuar en su derecho a la autodefinición, autodeterminación y soberanía de elección; analiza a la sociedad para enfocar programas de MS y evaluar los cambios de comportamientos y la ampliación de oportunidades para que las personas vivan el tipo de vida que llevan (Saunders et al., 2015).

Por su parte la Teoría de movimiento social vincula los encuadres y la acción colectiva para analizar los movimientos sociales (Daellenbach \& Parkinson, 2017) y el enfoque Meso-marketing se centra en los procesos de investigación y marketing que estudian simultáneamente al menos dos capas del mercado de cambio social y tiene como objetivo sintetizar micro y macro procesos para efectuar el cambio (House et al. , 1995).

Continuando con los niveles de influencia en los cambios de comportamientos el modelo social ecológico (SEM) Identifica la interrelación y la influencia de factores económicos, ambientales y sociales en la comunidad, intercultural e interpersonal e instituciones internas; bajo cuatro sistemas interrelacionados que interactúan entre sí ante los cambios de comportamiento deseados: I.Micro que encarnan aspectos de la identidad propia del individuo y su grupo social que afectan sus comportamientos, II.Meso que simboliza las estructuras sociales como leyes y comunicaciones de políticas gubernamentales para fomentar el desarrollo de un cambio de comportamiento III. Exo manifestada en la importancia de la comunidad en el desarrollo de la eficacia colectiva y IV.Macro personifican el contexto cultural del individuo así como sus expectativas culturales de la sociedad sobre el individuo que mitigan las barreras estructurales de infraestructura, políticas y la discriminación, este nivel supone que el público objetivo tiene la capacidad de comprender y cumplir con el comportamiento requerido (Lindridge et al., 2013). Posteriormente el Modelo Ecológico Conductual 
(BEM) de Bronfenbrenner, rescatado por Hovell et al. (2002) se enfoca en el comportamiento ecológico por cuanto entiende que el individuo está inmerso en un contexto permeado por la cultura y los valores, para encontrar barreras y facilitadores de cambios de comportamiento.

Este Modelo Ecológico Conductual (BEM) inicia comprendiendo las motivaciones y los comportamientos de las personas dentro de su sistema social incluyendo a todos los actores relevantes inclusive la industria (Brennan et al., 2015), porque los comportamientos de las personas están dentro de un sistema social y se explican por las influencias bidireccionales o multidireccionales que van desde la capa social (macro-sistema: contexto político y sociocultural), a través de capas comunitarias y locales (meso-sistemas: influencias sociales o facilitadores y conexiones entre actores) y al individuo en su microsistema, exo-sistema y el meso-sistema, repercutiendo en factores que influyen tanto en el individuo como en las entidades del sistema, por lo que se requiere la cooperación de varios interesados para crear soluciones sostenibles a los problemas sociales (Brennan et al., 2016).

En este sentido de unidad, la teoría de red de actores (ANT) sostiene que una red de actores humanos y no humanos se relacionan con intereses alineados entre los actores de redes temporales y dinámicas ancladas por otro actor individual o más amplio formando un colectivo, pero las redes pueden volverse inestables, con actores cambiantes y alianzas, por lo que su interés es qué reconocer las asociaciones fuertes y débiles en términos de poder (Gordon \& Gurrieri, 2014).

\section{CONCLUSIONES}

El MS ha evolucionado desde la difusión de los lineamientos de cambios de comportamientos, atravesando el proceso de planificación en los niveles de influencia hacia el individuo, sus grupos cercanos y las empresas, para llegar a los medios de comunicación digitales que pueden ayudar a los cambios de comportamiento (Dibb \& Carrigan, 2013).

Uno de los enfoques en tendencia es el Marketing Macro-Social entendido como el uso de MS, por parte de los gobiernos y otros actores intermedios dentro de un enfoque sistémico para generar un cambio social (Truong, 2017) se trata de influir en múltiples niveles y comportamientos sistémicos 
particularmente para problemas perversos (Kennedy y Parsons, 2014), pero hay tantos niveles interconectados de la sociedad involucrados que pensar en qué cambiar y en qué orden se vuelve abrumador (Kennedy, 2015), y surge el Marketing Social de Sistemas (SSM) argumentando que las iniciativas se deben abordar tres niveles juntos I. Macro (Gobierno, política y economía), II. Meso (sociedad) y III. Micro (creencias y hábitos individuales) (Kemper y Ballantine, 2017).

En una línea metodológica y totalmente diferente la teoría de la práctica social retomando los patrones de consumo proambientales en donde los cambios de comportamiento no se hacen educando 0 persuadiendo a las personas para que tomen decisiones diferentes, sino transformando las prácticas para hacerlas más sostenibles (Southerton et al., 2004). Esta metodología cualitativa enfocada en las prácticas sociales que rodean los procesos de consumo, más que en la toma de decisiones (Beatson et al., 2020), está convencida que las fuentes de comportamiento se radican en el desarrollo de las prácticas en sí mismas y no en la toma de decisiones (Shove y Warde, 2002), por lo que conecta las normas, valores e ideologías de la práctica (Hargreaves, 2011), (Shove y Pantzar, 2005) y convierte en el foco de análisis la práctica para rescatar el conocimiento tácito del hábito, la rutina involucrados en la actividad ( Meier et al. , 2018 ).

Resulta de interés en la literatura el análisis de los niños enfocando las intervenciones de MS en la efectividad a largo plazo de las intervenciones de MS para proporcionar habilidades y recursos que faciliten estilos de vida saludables en la edad adulta. Asi mismo, extender la implementación del MS a otros contextos como el acoso escolar, la seguridad cibernética, la seguridad contra incendios y la participación en la educación y continuar analizando las intervenciones, centrándose en el papel de los padres, maestros y otras partes interesadas que influyen en el desarrollo de los hábitos de vida saludables de los niños. Finalmente, existe una necesidad de establecer un consenso sobre las variables de resultado clave para las intervenciones de obesidad (Kubacki et al., 2015), (RussellBennett et al., 2013), (Domegan et al., 2016).

Por otra parte, desde la perspectiva empresarial en un entorno de Responsabilidad Corporativa, Sostenibilidad y Valor Compartido, el Modelo de Mercadeo Social con enfoque Sostenible (MSsS) se vincula para desarrollar una metodología en varios niveles de planificación, como se detalla en la Figura I. 
Figura I. Modelo de Mercadeo Social Corporativo con enfoque sostenible aplicable a grandes empresas en Colombia

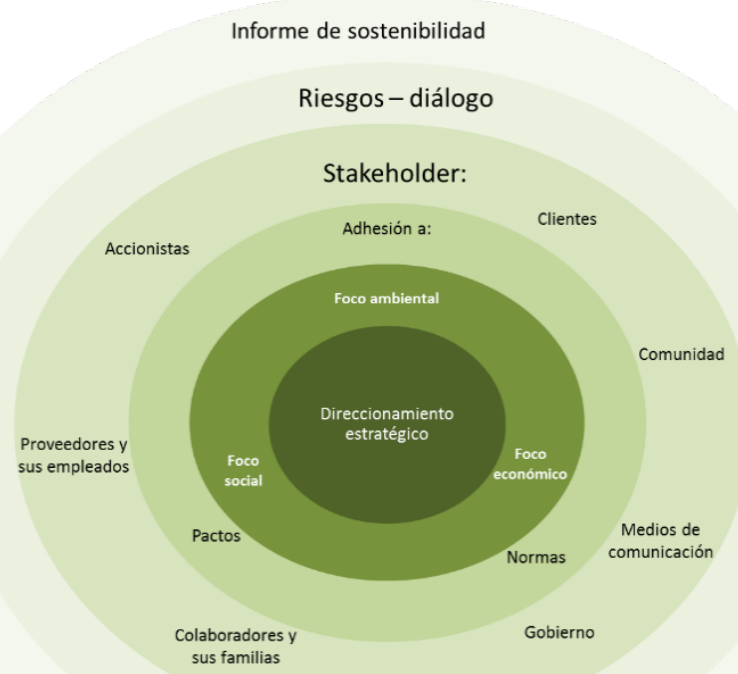

Global Reporting Initiative - GRI

(León y Lombana, 2015)

Desde el MS vinculado con Mercadeo de Servicios (Leo \& Zainuddin, 2017) se encuentra interesante crear entornos de servicio atractivos que faciliten el cambio de comportamiento o mantener dichos comportamientos, así como el rol del empleado del servicio público en la entrega de una experiencia de servicio efectiva para lograr resultados esperados. 0 la de la tecnología de autoservicio al bienestar social e individual, y la gestión de quejas en los programas de MS, la relación entre las políticas públicas y el pensamiento de servicio; las herramientas para auditar y gestionar el clima de servicio y los estándares en MS (Russell-Bennett et al., 2013) y los patrones de comportamiento de los usuarios después de que finalicen el uso del servicio (Domegan et al., 2016).

Existe una oportunidad para una teoría coherente del cambio en MS, que conecta el cambio de comportamiento con la transformación social de largo alcance (Domegan et al., 2016) como lo hace el Modelo MSsS para así vincular al MS y los Objetivos de Desarrollo Sostenible de las Naciones Unidas (Emerald, 2020). 
Lograr influencias más positivas en la comunidad en general y con el apoyo del cambio de políticas en torno al empaque y la educación para mejorar aún más las prácticas de consumo verde (Beatson et al., 2020).

Examinar los procesos de co-creación relacionados con el MS y el pensamiento sistémico y profundizar en el concepto de valor de comportamiento de French y Gordon (2015) para la co-creación con ciudadanos, partes interesadas y la sociedad. Así como investigar las brechas de co-creación de valor-acción y los enfoques de pensamiento crítico y frente al monitoreo de sistemas y el análisis de co-creación. Un aspecto fundamental de la expansión de la teoría y la práctica para el MS puede ser esta capacidad de medir y visualizar colectivamente la estructura de un sistema y su problema social compartido, y utilizar este conocimiento para diseñar estrategias a escala para la acción colectiva (Domegan et al., 2016).

\section{REFERENCIAS}

Andreasen, A. R. (1994). Social Marketing: Its Definition and Domain. Journal of Public Policy and Marketing, 13(1), 108-114. https://doi.org/10.2307/30000176

Andreasen, A. R. (1997). Prescriptions for Theory-Driven Social Marketing Research: A Response to Goldberg's Alarms. Journal of Consumer Psychology, 6(2), 189-196. https://doi.org/10.1207/s15327663jcp0602_04

Beatson, A., Gottlieb, U., \& Pleming, K. (2020). Green consumption practices for sustainability: an exploration through social practice theory. Journal of Social Marketing, 10(2), 197-213. https://doi.org/10.1108/JSOCM-07-2019-0102

Brennan, L., Fry, M. L., \& Previte, J. (2015). Strengthening social marketing research: Harnessing "insight" through ethnography. Australasian Marketing Journal, 23(4), 286-293. https://doi.org/10.1016/j.ausmj.2015.10.003

Brennan, L., Previte, J., \& Fry, M. L. (2016). Social marketing's consumer myopia: Applying a behavioural ecological model to address wicked problems. Journal of Social Marketing, 6(3), 219-239. https://doi.org/10.1108/JSOCM-12-2015-0079

Daellenbach, K., \& Parkinson, J. (2017). A useful shift in our perspective: integrating social movement thought into social marketing. Journal of Social Marketing, 7(2), 188-204. https://doi.org/10.1108/20426761211243937 
Domegan, C., Brychkov, D., Mchugh, P., Harkin, K., \& Fitzgerald, C. (2020). Marketing systems : A Listen, Learn , Leverage Framework. Journal of Macromarketing, 1-16. https://doi.org/10.1177/0276146720922282

Domegan, C., McHugh, P., Devaney, M., Duane, S., Hogan, M., Broome, B. J., Layton, R. A., Joyce, J., Mazzonetto, M., \& Piwowarczyk, J. (2016). Systems-thinking social marketing: conceptual extensions and empirical investigations. Journal of Marketing Management, 32(11-12), 11231144. https://doi.org/10.1080/0267257X.2016.1183697

Dibb S, Carrigan M (2013). Social marketing transformed: Kotler, Polonsky and Hastings reflect on social marketing in a period of social change. European Journal of Marketing. vol: 47 (9) pp: 1376-1398. https://doi.org/10.1108/EJM-05-2013-0248

Duffy, S., van Esch, P., \& Yousef, M. (2020). Increasing parental leave uptake: A systems social marketing approach. Australasian Marketing Journal, 28(2), 110-118. https://doi.org/10.1016/j.ausmj.2020.01.007

Fabbrizzi, S., Marinelli, N., Menghini, S., \& Casini, L. (2017). Why do you drink? A means-end approach to the motivations of young alcohol consumers. British Food Journal, 119(8), 18541869. https://doi.org/10.1108/BFJ-12-2016-0599

French,J., Gordon,R. (2015), Strategic Social Marketing, SAGE Publications, London Gurrieri L Previte J Brace-Govan J (2013). Women's Bodies as Sites of Control: Inadvertent Stigma and Exclusion in Social Marketing. Journal of Macromarketing, 3-9. https://doi.org/10.1177/0276146712469971

Gordon R Gurrieri L (2014). Towards a reflexive turn: Social marketing assemblages. Journal of Social Marketing. 261-278. https://doi.org/ 10.1108/JSOCM-02-2014-0015

Hargreaves, T. (2011), "Practicing behavior change: applying social practice theory to proenvironmentalbehaviorchange", Journal of Consumer Culture, Vol.11 No.1, pp.79-99.

House, R., Rousseau, D.M. and Thomas-Hunt, M. (1995), "The meso paradigm: aframework for integration of mcro and macro organizational", in Cummings, L.L, and Staw, B (Eds), Research in Organizational Behaviour, JAI Press, Greenwich, CT, Vol.17, pp. 71-114

Hovell, M.F., Wahlgren, D.R. and Gehrman, C.A. (2002), "The behavioral ecological model”, in DiClemente, R.J, Crosby, R.A. and Kegler, M.C. (Eds), Emerging Theories in Health Promotion Practice and Research: Strategies for Improving Public Health, John Wiley and Sons, SanFrancisco,pp.347-385. 
Kemper J Ballantine P. (2020) Targeting the structural environment at multiple social levels for systemic change: The case of climate change and meat consumption. Journal of Social Marketing. 2020 vol: 10 (1) pp: 38-53. https://doi.org/10.1108/JSOCM-02-2019-0024

Kennedy, A. M. (2015). Macro-social marketing. Journal of Macromarketing, 35(4), 1-12. doi:10.1177/0276146715617509

Kennedy, A. M., \& Parsons, A. (2014). Social engineering and social marketing: Why is one good and the other bad. Journal of Social Marketing, 4(3), 198-209. doi:10.1108/jsocm-01-20140006

Kotler, P., \& Lee, N. (2004). Best of Breed 2016. Stanford Social Innovation Review, 13-23. http://c4lpt.co.uk/top100tools/best-of-breed/

Kotler, P., \& Zaltman, G. (1971). Social Marketing : An Approach To Planned Social Change. Journal of Marketing, 35(July), 3-12. https://doi.org/10.2307/1249783

Kotler, P. and Roberto, E. (1989),Social Marketing: Strategies for Changing Public Behavior , The Free Press, New York, NY.

Kubacki, K., Rundle-Thiele, S., Lahtinen, V., \& Parkinson, J. (2015). A systematic review assessing the extent of social marketing principle use in interventions targeting children (2000-2014). Young Consumers, 16(2), 141-158. https://doi.org/10.1108/YC-08-2014-00466

Meier,P.S., Warde,A. and Holmes,J. (2018), "All drinking is not equal: how a social practice theory lens could enhance public health research on alcohol and other health behaviours", Addiction, Vol.113No.2,pp.206-213.

Lazer W (1969) Marketing's Changing Social Relationships Author(s):: Journal of Marketing. 3-9. American Marketing Association. http://www.jstor.org/stable/1248739

Leo, C., \& Zainuddin, N. (2017). Exploring value destruction in social marketing services. Journal of Social Marketing, 7(4), 405-422. https://doi.org/10.1108/JSOCM-03-2017-0022

León A Lombana J. (2015). Modelo de Mercadeo Social con enfoque sostenible aplicable a grandes empresas en Colombia. Acacia, 1-30. http://acacia.org.mx/busqueda/pdf/MODELO_DE_MERCADEO_SOCIAL_CON_ENFOQUE_SO STENIBLE_APLICABLE_EN_GRANDES_EMPRESAS_EN_COLOMBIA.pdf

Lindridge, A., MacGaskill, S., Ginch, W., Eadie, D., \& Holme, I. (2013). Applying an ecological model to social marketing communications. European Journal of Marketing, 47(9), 1399-1420. https://doi.org/10.1108/EJM-10-2011-0561

Russell-Bennett, R., Wood, M., \& Previte, J. (2013). Fresh ideas: Services thinking for social 
marketing. Journal of Social Marketing, 3(3), 223-238. https://doi.org/10.1108/JSOCM-02-20130017

Saunders S Barrington D Sridharan S (2015),"Redefining social marketing: beyond behavioural change", Journal of Social Marketing, Vol. 5 Iss 2 pp. -Permanent link to this document: http://dx.doi.org/10.1108/JSOCM-03-2014-0021

Southerton D., Chappells Ch., Vliet B., V. (2004) Sustainable Consumption: The Implications of Changing Infrastructures of Provisión. Ed. Edward Elgar.

Shove, E. and Pantzar, M. (2005), "Consumers, producers and practices: understanding the invention and reinvention of Nordicwalking", Journal of Consumer Culture,Vol.5 No.1,pp.43-64.

Shove, E. and Warde, A. (2002), "Inconspicuous consumption: the sociology of consumption, lifestyles and the environment", Sociological Theory and the Environment: Classical Foundations, Contemporary Insights, Vol.230 No.51.

Truong, V. D (2017). Government-led macro-social marketing programs in Vietnam: Outcomes, challenges, and implications. Journal of Macromarketing, 37, 409-425. 